\title{
LAS HORMIGAS (HYMENOPTERA, FORMICIDAE) ASOCIADAS A PULGONES (HEMIPTERA, APHIDIDAE) EN LA PROVINCIA DE VALENCIA
}

\author{
V. A. Suay-Cano *, A. Tinaut ** y J. Selfa *
}

\begin{abstract}
RESUMEN
Se han recogido veintiséis especies de hormigas asociadas a pulgones, pertenecientes a doce géneros. De las tres subfamilias identificadas, Formicinae ha sido la más representada en cuanto a número de muestras, géneros y especies. Lasius niger, por otra parte, ha sido la especie que se ha encontrado junto a un mayor número de pulgones (cincuenta y ocho especies), demostrando ser la hormiga con mayor capacidad para establecer asociaciones con las diferentes especies de pulgones.

Se han establecido un total de 224 asociaciones diferentes entre las hormigas y los áfidos, de las cuales 164 no se han encontrado citadas en la bibliografía consultada para España.

Palabras claves: pulgones, hormigas, relaciones hormiga-pulgón, Valencia, España.
\end{abstract}

\section{ABSTRACT}

The ants (Hymenoptera, Formicidae) associated with aphids (Hemiptera, Aphididae) in the province of Valencia

Twenty-six species of ants associated with aphids, belonging to twelve genera, are collected. Three subfamilies are identified, being the Formicinae the more represented about number of samples, genera and species. On the other hand, Lasius niger has been the species that is found with a more number of aphids (fifty eight species), and it seems to be the ant with a more capacity to establish associations with the different species of aphids.

224 different associations between the ants and the aphids are established and 164 of them are not recorded in the bibliography consulted for Spain.

Key words: aphids, ants, ant-aphid relationships, Valencia, Spain.

\section{Introducción}

Las hormigas constituyen uno de los grupos de insectos más ampliamente estudiado como consecuencia de su abundancia y de la repercusión del grupo en diferentes campos de la biología (Hölldobler y Wilson, 1990). Uno de los aspectos clásicamente conocido es el de las relaciones que mantienen con los pulgones, de las que aún quedan numerosos detalles por conocer. La banalidad de estas relaciones ha llevado a la creencia de que están generalizadas para todas las especies de hormigas, sin embargo ni todas las especies de hormigas son afidófilas, ni todas las especies de áfidos son mirmecófilas. Incluso en el caso de especies afidófilas o mirmecófilas, las relaciones hormiga-

* Dpto. de Biología Animal (Entomología), Facultad de Biología. Universidad de Valencia, C/ Dr. Moliner, 50, 46100 Burjassot (Valencia).

** Dpto. de Biología Animal y Ecología, Facultad de Ciencias. Universidad de Granada, Campus Universitario de Fuentenueva, 18071 Granada. 
áfido están reguladas a diferentes niveles (Sudd, 1987). Uno de los primeros aspectos a conocer en estas relaciones es el establecimiento de las especies, de hormigas y pulgones, que muestran afinidad entre sí. En este sentido, el inventario es aún muy escaso y muy fragmentado.

En general la mayoría de los trabajos sobre las relaciones entre estos dos grupos se refieren a especies concretas (Evans y Leston, 1971; Pontin, 1978; Delfino y Buffa, 2000, entre otros) o a aspectos generales en este tipo de relaciones (Sudd, 1987; Bristow, 1991a y b; Sakata, 1994), siendo escasos los estudios referidos al total de especies en un área o hábitat concreto. Este tipo de estudios es de gran interés porque permite testar el nivel de plasticidad específica en las relaciones hormiga-áfido.

En España, pese a que hay numerosos trabajos publicados tanto de áfidos (Mier Durante, 1978; González Funes, 1987; Tizado Morales, 1990; Nieto Nafría et al., 1991; Núñez Pérez, 1991; Suay Cano, 1996, entre otros) como de formícidos (Collingwood, 1976; López, 1991a; Tinaut,1991, entre otros), el conocimiento de las relaciones entre estos dos grupos de insectos es más bien escaso (Retana et al., 1987; Tizado Morales, 1990; Núñez Pérez, 1991; Tizado et al., 1993), y se basa principalmente en estudios realizados en la provincia de León, de condiciones climáticas diferentes a las del área de estudio del presente trabajo, la provincia de Valencia.

Como resultado del estudio realizado sobre la afidofauna de la provincia de Valencia, y de algunos grupos de insectos asociados a los pulgones, se exponen en este artículo los resultados obtenidos acerca de las especies de hormigas encontradas en relación con los áfidos.

\section{Material y Métodos}

La recolección de hormigas ha ido ligada a la de las colonias de pulgones con los que se pretenden relacionar. En plantas donde los pulgones se ocultan entre las brácteas, entre las flores o enterrados, o cuando su color los hace crípticos, el detectar hormigas moviéndose de un lugar a otro puede indicarnos la localización de la colonia de áfidos.

La captura de hormigas puede resultar complicada, si tenemos en cuenta que se trata, como es bien conocido, de insectos verdaderamente ágiles y en ocasiones agresivos, sobre todo si las comparamos con los áfidos, normalmente poco ágiles y bastante pasivos. Así, pese a recoger las muestras de hormigas de la misma forma que se hace con los pulgones (con la ayuda de un pincel y un frasco de alcohol), se ha de tener mayor cuidado y precisión, ya que, incluso, se pueden desperdiciar algunas muestras de pulgones al moverse las hormigas rápidamente de un sitio para otro. Es recomendable capturarlas en primer lugar para evitar que al tratar de recoger los pulgones, aquéllas reaccionen con rápidos movimientos y escapen o caigan al suelo. Una vez capturadas, éstas se introducen en tubos de alcohol de $70 \%$ junto a los pulgones de la colonia y se transportan al laboratorio, donde se realiza la separación de los dos grupos y se procede a la identificación de las especies. Cada conjunto de pulgones y hormigas constituyen una muestra, por lo que de una misma planta se pueden obtener varias muestras.

En total, se han tomado muestras en 85 localidades, pertenecientes a todas las comarcas en que se divide la provincia de Valencia (muestras que se encuentran depositadas en el Laboratorio de Entomología de la Facultad de Biología de la Universidad de Valencia). En el Apéndice I se da el listado de localidades muestreadas, ordenadas alfabéticamente dentro de cada comarca, asignándoles un número de orden, e indicándose, a su vez, la altura y las coordenadas U.T.M.

\section{Resultados}

A continuación se expone la relación de especies de hormigas que se han encontrado asociadas a pulgones. No siempre existe la total seguridad de que las hormigas se encuentren atendiendo a los áfidos; así, en ocasiones, probablemente, únicamente se hallaban junto a éstos para alimentarse de la melaza que queda depositada sobre las hojas. En ningún caso, aunque es un hecho constatado que se puede producir (Sakata, 1994), se han encontrado hormigas predando sobre los pulgones.

La relación de las especies encontradas se realiza por orden alfabético, partiendo de la división en subfamilias, subdivididas éstas a su vez en tribus. Se indica, para cada formícido, los pulgones junto a los que se ha hallado, las plantas sobre las que se encontraban ambos y la localidad, mediante el número de orden que se le ha adjudicado en el Apéndice I, y fecha de recolección de la muestra. Cuando algún pulgón no se haya podido determinar se reseñará como "indeterminado" al final del listado.

Por otra parte, queremos reseñar que a algunas especies de pulgones que no se han podido determinar a nivel específico, no por encontrarse el 
material en malas condiciones o ser especialmente dificultosa su identificación, si no por no parecer pertenecer a ninguna de las especies conocidas, se les ha asignado un número de orden. Así aparecen en el texto tres especies: Aphis (A.) sp.1, Aphis (A.) sp.2 y Dysaphis (D.) sp.1. Las dos primeras corresponden respectivamente a Aphis (A.) sp.3 y Aphis (A.) sp.5 (en Suay Cano, 1996) y la tercera, a Dysaphis (D.) sp. ined. del mismo trabajo.

Al final del trabajo, tras el Apéndice I, damos la lista de especies de pulgones y las correspondientes especies de hormigas encontradas con ellos (Apéndice II). En dicho apéndice, se indican con un asterisco (*) aquellas asociaciones que no se han encontrado descritas en la literatura consultada para España.

\section{Dolichoderinae: Dolichoderini}

\section{Dolichoderus (Hypoclinea) quadripunctatus (Linné, 1771)}

Es una hormiga típicamente arborícola y de alimentación omnívora, que vive en pequeñas sociedades bajo la corteza, agallas o ramas muertas de árboles no resinosos. Aparece en casi toda la región mediterránea y es la única especie del género que vive en Europa.

Panaphis juglandis Frisch, 1734: Juglans regia L. (6:16.8.1991).

Se ha encontrado junto a Crematogaster scutellaris, Camponotus (C.) fallax y Lasius niger.

\section{Dolichoderinae: Tapinomini}

\section{Linepithema humile (Mayr, 1868)}

De alimentación típicamente omnívora, aprovecha la melaza producida por algunos insectos, así como otras sustancias azucaradas. Se trata de una especie muy eficaz en el cuidado y protección de pulgones, pues ataca y desplaza a las larvas de neurópteros y de sírfidos (Diptera), si bien raramente molesta a los coccinélidos (Bristow, 1991b).

Es una hormiga de origen sudamericano, probablemente de Brasil, que en la actualidad es cosmopolita. Presenta una distribución litoral, siendo muy común en lugares de influencia humana. Se ha recogido de forma muy abundante, como era de esperar por lo comentado sobre su distribución, en la Devesa de la Albufera (22 muestras), donde ha sido la única especie encontrada. También se ha recogido, bastante abundante, en el Jardín Botánico de Valencia, situado en el interior de la ciudad (14 muestras), sin encontrar, como en el caso del paraje anterior, ninguna otra especie compartiendo la atención de los pulgones.

En una ocasión se ha observado cómo una ninfa de Aphis (A.) confusa era transportada en las mandíbulas de una hormiga.

Acyrthosiphon (A.) loti (Theobald, 1913): Anthyllis cytisoides L. (54:29.5.1989). Anuraphis subterranea (Walker, 1852): Thapsia villosa L. (54:23.6.1989). Aphis (A.) confusa Walker, 1849: Scabiosa atropurpurea L. (54:26.5.1989). Aphis (A.) craccivora Koch, 1854: Anthyllis cytisoides L. (54:29.5.1989), Dorycnium pentaphyllum Scop. ssp. gracile (Jordan) Rouy (54:26.5.1989), Medicago sp. (9:9.5.1991), Opuntia spp. (58:14.6.1988). Aphis (A.) cytisorum Hartig, 1841: Dorycnium hirsutum (L.) Ser. (54:7.6.1989). Aphis (A.) fabae Scopoli, 1763: Carduus pycnocephalus L. (79:4.6.1991), Malvaceae (58:9.5.1987), Philadelphus verrucosus Schrad. (58:17.5.1988), Smyrnium olusatrum L. (58:30.3.1988), Solanum nigrum L. (54:17.10.1990/13.11.1990), Urtica dioica L. (39:7.5.1991), Yucca aloifolia L. (58:4.5.1988). Aphis (A.) fabae fabae Scopoli, 1763: Carduus pycnocephalus L. (21:29.4.1990), Foeniculum vulgare Miller ssp. piperitum (Ucria) Coutinho (54:17.10.1990), Rubia peregrina L. (30:18.4.1991), Silybum marianum (L.) Gaertner (79:15.5.1990). Aphis (A.) frangulae frangulae Kaltenbach, 1845: Rhamnus alaternus L. (54:31.7.1989/ 17.10.1990/13.11.1990), Rhamnus oleoides L. ssp. angustifolia (Lange) Rivas Goday et Rivas-Martínez (52:26.5.1989, 54:31.7.1989). Aphis (A.) frangulae gossypii Glover, 1877: Aeonium sp. (58:4.5.1988), Ajuga iva (L.) Schereber (70:8.5.1990), Conyza albida Willd. ex Spreng. (54:17.10.1990), Helichrysum stoechas (L.) Moench (54:29.5.1989), Hibiscus syriacus L. (58:7.10.1987), Malvaceae (58:9.5.1987), Philadelphus spp. (58:27.4.1987), Senecio petasitis DC. (58:1.6.1987), Smilax aspera L. (54:31.7.1989), Thapsia villosa L. (54:23.6.1989), Yucca aloifolia L. (58:4.5.1988). Aphis (A.) hederae Kaltenbach, 1843: Hedera helix L. (37:23.5.1991, 58:15.10.1986/29.2.1988). Aphis (A.) helianthemi Ferrari, 1872: Helianthemum syriacum (Jacq.) Dum.-Cours. (54:26.5.1989). Aphis (A.) nasturtii Kaltenbach, 1843: Lavatera cretica L. (71:8.5.1990). Aphis (A.) pilosellae (Börner, 1952): Picris echioides L. (39:7.5.1991). Aphis (A.) pomi De Geer, 1773: Malus domestica Borkh. (37:12.6.1990). Aphis (A.) ruborum (Börner, 1932): Rubus ulmifolius Schott (39:7.5.1991). Aphis (A.) sp.: Anagallis arvensis L. (27:30.4.1991), Inula viscosa (L.) Aiton (54:17.10.1990), Scrophularia canina L. (70:8.5.1990), Seseli tortuosum L. (54:17.10.1990). Aphis (A.) spiraecola Patch, 1914: Arbutus unedo L. (46:10.11.1988), Conyza albida Willd. ex Spreng. (54:17.10.1990), Fuchsia magellanica Lam. (58:18.5.1988), Hibiscus syriacus L. (58:7.10.1987), Malvaceae (58:9.5.1987), Oenanthe lachenalii C. C. Gmelin (51:25.4.1991), Philadelphus spp. (58:27.4.1987), Pittosporum tobira (Thumb.) Aiton Fil. (58:15.10.1986), Indeterminada (58:27.4.1987). Aphis (A.) tirucallis Hille Ris Lambers, 1954: Euphorbia terracina L. (54:11.4.1990). Aphis (A.) umbrella (Börner, 1950): Lavatera cretica L. (21:29.4.1990), Malva L. (70:3.4.1990), Malva sylvestris L. (20:3.5.1990). Aphis (A.) verbasci Schrank, 1801: Verbascum boerhavii L. (54:23.4.1991). 
Aphis (Protaphis) terricola Rondani, 1847: Centaurea seridis L. (54:23.4.1991). Brachycaudus (Acaudus) cardui (L., 1758): Carduus tenuiflorus Curtis (21:30.4.1991,51:25.4.1991), Cynara scolymus L. (30:20.3.1992). Brachycaudus (Acaudus) sp.: Senecio spp. (46:25.4.1991). Chaitophorus leucomelas Koch, 1854: Populus nigra L. (37:23.5.1991/ 9.7.1991, 70:3.5.1991). Chaitophorus populeti (Panzer, 1801): Populus alba L. (50:14.4.1991, 54:28.6.1989,70:8.5.1990/ 3.5.1991), Populus alba L. ssp. bolleana Lauche (58:18.5.1988). Chaitophorus sp.: Populus nigra L. (39:7.5.1991,46:10. 11.1988). Cinara (Cupressobium) tujafilina (Del Guercio 1909): Thuja orientalis L. (58:29.3.1988). Dysaphis (D.) sp.: Foeniculum vulgare Miller ssp. piperitum Ucria (Coutinho) (37:12.6.1990). Hyalopterus pruni (Geoffroy, 1762): Arundo donax L. (54:23.4.1991), Phragmites australis (Cav.) Trin ex Steudel (51:31.5.1990). Macrosiphum (M.) euphorbiae (Thomas, 1878): Lavatera cretica L. (71:8.5.1990). Melanaphis bambusae (Fullaway, 1910): Phyllostachys nigra (Lodd.) Munro (58:9.5.1987/30.3.1988). Melanaphis donacis (Passerini, 1862): Arundo donax L. (50:11.4.1991,70:8.5.1990). Myzocallis (M.) coryli (Goetze, 1778): Corylus cornuta Marsh. (58:31.5.1988) Rhopalosiphum padi (L., 1758): Bromus unioloides Kunth (52:22.5.1989). Tetraneura (T.) ulmi (L., 1758): Zea mays L. (37:12.6.1990). Thelaxes suberi (Del Guercio, 1911): Quercus coccifera L. (54:7.6.1989/17.10.1990/ 13.11.1990). Toxoptera aurantii (Boyer de Fonscolombe, 1856): Erica multiflora L. (47:4.4.1991), Philadelphus verrucosus Schrad. (58:17.5.1988), Rhamnus rhostornii E. Pritz. (58:8.11.1988), Zanthoxylum alatum Steud. (58:8.11.1988), Zanthoxylum pterota H. B. et K. (58:24.5.1988). Indeterminado: Eucalyptus sp. (54:7.6.1989).

Tapinoma erraticum (Latreille, 1798)

Se trata de una especie muy combativa que vive preferentemente sobre terrenos rocosos calcáreos soleados o sobre arenas muy permeables. Se distribuye por toda Europa y por Asia occidental y central.

Aphis (A.) craccivora Koch, 1854: Lepidium draba L. (36:24.5.1990). Aphis (A.) fabae Scopoli, 1763: Iris pseudacorus L. (46:14.4.1988). Aphis (A.) ruborum (Börner, 1932): Rubus ulmifolius Schott (79:14.5.1991). Aphis (A.) sp.: Malva sylvestris L. (20:30.4.1991), Indeterminada (9:9.5.1991). Cinara (Cupressobium) juniperi (De Geer, 1773): Juniperus oxycedrus L. (15:5.6.1990).

\section{Tapinoma nigerrimum (Nylander, 1886)}

Construye profundos nidos en el suelo, adaptándose bien a suelos arcillosos y regados, soportando incluso inundaciones. Parece ser originaria de Marruecos; se distribuye por toda la región mediterránea, así como por Asia central.

Aunque en principio prácticamente no sobrepasa cotas superiores a los 800 metros, a diferencia de la especie anterior (Bernard, 1968), Tizado (1990) la cita cerca de los 1.000 metros de altitud. En este trabajo se ha recogido, en 32 de las 57 muestras, de varias localidades por encima de 800 metros, dos de dichas localidades por encima de 1.000 metros (Ahillas, 870 metros, Higueruelas, 870 metros, Puebla de San Miguel, 1.100 metros, Sinarcas, 900 metros, Vallanca, 950 metros, y la Yesa, 1.008 metros).

Acyrthosiphon (A.) daphnidis Ilharco, 1996: Daphne gnidium L. (12:20.4.1990). Aphis (A.) brotericola Mier, 1978: Euphorbia esula L. (18:8.6.1991), Euphorbia segetalis L. (4:6.6.1991/27.6.1991/18.7.1991). Aphis (A.) brunnea Ferrari, 1872: Ononis spp. (4:6.6.1991). Aphis (A.) cisticola Leclant et Remaudière, 1972: Cistus albidus L. (15:28.6.1990). Aphis (A.) craccivora Koch, 1854: Dorycnium hirsutum (L.) Ser. (15:11.7.1991), Genista scorpius (L.) DC. (12:30.5.1991), Medicago sativa L. (4:27.6.1991,15:25.6.1991), Vicia sativa L. (9:9.5.1991). Aphis (A.) fabae Scopoli, 1763: Carduus assoi (Willk.) Pau (7:11.6.1991), Carpobrotus edulis (L.) N. E. Br. (28:31.5.1990), Centaurea aspera L. (37:10.7.1991), Echium plantagineum L. (81:14.5.1991), Foeniculum vulgare Miller ssp. piperitum (Ucria) Coutinho (81:14.5.1991), Rumex crispus L. (37:10.7.1991). Aphis (A.) fabae fabae Scopoli, 1763: Eryngium campestre L. (15:25.6.1991). Aphis (A.) fabae solanella Theobald, 1914: Rumex crispus L. (35:28.5.1991). Aphis (A.) nasturtii Kaltenbach, 1843: Mentha longifolia (L.) Hudson (1:2.7.1991), Rumex crispus L. (35:28.5.1991). Aphis (A.) sedi Kaltenbach, 1843: Sedum sediforme (Jacq.) Pau (65:5.7.1990). Aphis (A.) serpylli Koch, 1854: Thymus vulgaris L. (4:27.6.1991). Aphis (A.) sp.: Melilotus albus Medicus (6:18.7.1991). Aphis (A.) sp.1: Rosmarinus offinalis L. (15:25.6.1991). Aphis (A.) tirucallis Hille Ris Lambers, 1954: Euphorbia flavicoma DC. $(35: 28.5 .1991)$. Aphis (A.) umbrella (Börner, 1950): Malva alcea L. (32:28.5.1991). Aphis (A.) verbasci Schrank, 1801: Verbascum sinuatum L. (65:18.6.1991). Aphis (A.) vitalbae Ferrari, 1872: Clematis flammula L. (14:17.7.1991). Aphis (Protaphis) terricola Rondani, 1847: Centaurea salmantica L. (6:19.6.1990), Centaurea seridis L. (28:18.4.1991). Brachycaudus (Acaudus) cardui (L., 1758): Carduus assoi (Willk.) Pau (15:28.6.1990), Carduus tenuiflorus Curtis (19:26.6.1990,32:28.5.1991), Silybum marianum (L.) Gaertner (24:8.6.1989). Brachycaudus (Appelia) tragopogonis (Kaltenbach, 1843): Scorzonera angustifolia L. (19:26.6.1990). Brachyunguis harmalae Das, 1918: Carpobrotus edulis (L.) N. E. Br. (28:18.4.1991). Cavariella (C.) aegopodii (Scopoli, 1763): Foeniculum vulgare Miller ssp. piperitum (Ucria) Coutinho (81:14.5.1991). Chaitophorus leucomelas Koch, 1854: Populus nigra L. (30:18.4.1991). Cinara (C.) maritimae (Dufour, 1833): Pinus sp. (15:11.7.1991), Pinus sylvestris L. (18:3.7.1990). Cinara (Cupressobium) juniperi (De Geer, 1773): Juniperus oxycedrus L. (15:28.6.1990). Cinara (Cupressobium) tujafilina (Del Guercio, 1909): Cupressus sempervirens L. (16:7.6.1990). Cinara sp.: Juniperus communis L. (15:11.7.1991), Juniperus oxycedrus L. (4:27.6.1991), Pinus halepensis Miller (4:27.6.1991). Dysaphis (D.) apiifolia petroselini (Börner, 1950): Foeniculum vulgare Miller ssp. piperitum (Ucria) Coutinho (81:4.6.1991). Dysaphis (D.) sp.1: Eryngium campestre L. (15:25.6.1991). Dysaphis (Pomaphis) plantaginea (Passerini, 1860): Malus domestica Borkh. (15:11.7.1991). Macrosiphoniella (M.) helichrysi Remaudière, 1952: Helichrysum serotinum Boiss. (18:28.6.1990). Rhopalosiphum padi (L., 1758): Festuca valentina (St.-Ives) Markgr.-Dannenb (35:28.5.1991). Sipha (Rungsia) maydis Passerini, 1860: 
Bromus L. (28:18.4.1991). Thelaxes suberi (Del Guercio, 1911): Quercus ilex L. (4:27.6.1991). Indeterminado: Cichorium intybus L. (37:10.7.1991). Indeterminado: Digitalis obscura L. (4:27.6.1991). Indeterminado: Pinus sylvestris L. $(15: 11.7 .1991)$

Se ha encontrado una vez junto a Formica subrufa, atendiendo a Aphis (A.) brotericola sobre Euphorbia segetalis (4:6.6.1991), dos veces junto a Lasius niger, atendiendo a Aphis (A.) fabae solanella y a Aphis (A.) nasturtii, ambas sobre Rumex crispus (35:28.5.1991), y en otra ocasión, junto a Crematogaster sordidula, atendiendo a Aphis (A.) sp. 1 .

\section{Formicinae: Camponotini}

Camponotus (C.) fallax (Nylander, 1856)

Esta especie nidifica bajo las piedras, en ramas caídas y bajo la corteza de los árboles, en sociedades poco numerosas. Es bastante común en toda Europa.

Bernard (1968) indica que algunos autores consideran a esta especie como una variedad de Camponotus (Myrmentoma) caryae (Fitch, 1855), que parece ser típicamente arborícola, encontrándose frecuentemente sobre nogales. Se ha de desechar esta idea pues se trata de subgéneros diferentes. Tomándolo como coincidencia, se puede decir que Camponotus (C.) fallax se ha recogido también sobre nogal, y se puede suponer, ya que no se han encontrado datos al respecto en la bibliografía, que se trata de una especie arborícola.

Panaphis juglandis Frisch, 1734: Juglans regia L. (6:16.8.1991).

Se ha encontrado junto a Crematogaster scutellaris, Dolichoderus (Hypoclinea) quadripunctatus y Lasius niger.

\section{Camponotus (Myrmentoma) lateralis (Olivier, 1791)}

Se trata de una especie típicamente arborícola (Tinaut, 1991) que en este trabajo, sin embargo, se ha encontrado sobre plantas herbáceas en tres ocasiones, y únicamente una vez sobre árboles (nogal). Nidifica en ramas secas y bajo la corteza de los árboles, en sociedades poco numerosas. Es de alimentación omnívora y busca muy a menudo pulgones. Común sobre todo en la región mediterránea, hasta Asia Menor.

Aphis (A.) craccivora Koch, 1854: Sedum sediforme (Jacq.) Pau (64:20.6.1991). Aphis (A.) helianthemi Ferrari, 1872:
Helianthemum syriacum (Jacq.) Dum.-Cours. (14:17.7.1991). Nearctaphis bakeri (Cowen, 1895): Trifolium pratense L. (6:18.7.1991). Panaphis juglandis Frisch, 1734: Juglans regia L. (6:6.6.1991).

Se ha encontrado junto a Plagiolepis pygmaea atendiendo a Nearctaphis bakeri.

\section{Camponotus (Myrmentoma) piceus (Leach, 1825)}

Vive preferentemente en suelos arenosos o arcillosos de lugares abiertos, y sólo rara vez se adentra en los bosques. Se encuentra ampliamente distribuida por Europa, y se ha encontrado también en el norte de África.

Aphis (A.) craccivora Koch, 1854: Cytisus heterochrous Webb ex Colmeiro (35:7.6.1990), Genista scorpius (L.) DC. (4:27.6.1991). Aphis (A.) fabae Scopoli, 1763: Carduus assoi (Willk.) Pau (15:5.6.1990). Cinara (C.) maritimae (Dufour, 1833): Pinus nigra Arnold ssp. salzmannii (Dunal) Franco (4:13.7.1990). Cinara (Cupressobium) cupressi (Buckton, 1881): Juniperus phoenicia L. (15:5.6.1990). Cinara (Cupressobium) juniperi (De Geer, 1773): Juniperus communis L. ssp. hemisphaerica (K. Presl) Nyman (4:24.6.1989).

Se ha encontrado junto a Myrmica scabrinodis en dos ocasiones, una atendiendo a Cinara (C.) maritimae y la otra a Aphis (A.) craccivora sobre Genista scorpius.

\section{Camponotus (Myrmosericus) cruentatus (Latreille, 1802)}

Se alimenta principalmente de la melaza de los áfidos; también se alimenta de otras fuentes azucaradas $\mathrm{y}$, en menor proporción, captura presas, normalmente artrópodos. Defiende con agresividad contra otras hormigas sus fuentes de alimentos y se han observado combates con otra especie que atiende pulgones, Camponotus (Tanaemyrmex) sylvaticus (Alsina et al., 1988). Nidifica bajo grandes piedras en los bosques, casi siempre en suelos calizos. Es una especie de origen ibero-mauritano, común en la región mediterránea.

Aphis (A.) craccivora Koch, 1854: Anthyllis cytisoides L. (24:29.5.1991). Aphis (A.) fabae fabae Scopoli, 1763: Papaver somniferum L. ssp. setigerum (DC.) Arcangeli (24:29.5.1991).

Camponotus (Tanaemyrmex) aethiops (Latreille, 1798)

Es una especie que construye siempre los nidos en el suelo, sobre todo en los de tipo calizo. Es bastante común en la región mediterránea.

Aphis (A.) craccivora Koch, 1854: Medicago sativa L. (64:20.6.1991). Aphis (A.) fabae Scopoli, 1763: Cirsium arvense (L.) Scop. (4:27.6.1991). Aphis (A.) frangulae gossypii Glover, 1877: Labiatae (6:6.6.1991). Brachycaudus (Acaudus) 
cardui (L., 1758): Carduus tenuiflorus Curtis (1:6.6.1991). Cinara sp.: Pinus halepensis Miller (4:27.6.1991).

Se ha encontrado, atendiendo a Aphis (A.) frangulae gossypii, junto a Lasius niger.

\section{Camponotus (Tanaemyrmex) foreli Emery, 1881}

Los nidos se sitúan bajo las piedras y están formados por pocos individuos. Esta especie, de hábitos diurnos y termófila, se alimenta fundamentalmente del néctar de las flores, pero también de la melaza de los áfidos; la captura de presas es muy rara (Retana et al., 1987). Según estos autores parece preferir a Aphis (A.) fabae frente a otros pulgones como Brachycaudus (Acaudus) cardui, Cinara (C.) maritimae y Eulachnus tuberculostemmatus (Theobald, 1915), intensamente atendidos por otras hormigas. Se trata de una hormiga no agresiva que no defiende sus fuentes de alimento, por lo que coexiste con otras especies. Es muy común en el área mediterránea.

Aphis (A.) craccivora Koch, 1854: Leguminosae (60:20.6.1991). Aphis (A.) frangulae gossypii Glover, 1877: Phlomis lychnitis L. (60:20.6.1991).

Camponotus (Tanaemyrmex) sylvaticus (Olivier, 1791)

Se trata de una especie de hábitos principalmente crepusculares y nocturnos (Alsina et al., 1988), que habita principalmente en los bosques, construyendo nidos más poblados y menos profundos que los de Camponotus (Tanaemyrmex) aethiops. Atiende pulgones, entre otras fuentes de alimentos, y se trata de una especie bastante agresiva, manteniendo combates con Camponotus (Myrmosericus) cruentatus, como ya hemos mencionado. Parece ser originaria de África del Norte, y se distribuye por el oeste europeo.

Aphis (A.) fabae fabae Scopoli, 1763: Eryngium campestre L. (76:4.6.1991), Rumex sp. (76:4.6.1991). Aphis (A.) frangulae gossypii Glover, 1877: Lavatera maritima Gouan (31:31.5.1990). Aphis (A.) ulicis Walker, 1870: Ulex parviflorus Pourret (76:4.6.1991). Brachycaudus (Acaudus) cardui (L., 1758): Carduus pycnocephalus L. (57:7.5.1991). Cinara (C.) maghrebica Mimeur, 1934: Pinus halepensis Miller (33:5.7.1990,76:14.5.1991/4.6.1991). Lachnus roboris (L., 1758): Quercus coccifera L. (33:5.7.1990). Indeterminado: Rhamnus alaternus L. (73:22.5.1990).

Colobopsis truncatus (Spinola, 1808)

Se trata de una especie típicamente arborícola (Tinaut, 1991), que vive principalmente sobre árbo- les no resinosos; fundamentalmente sobre robles, aunque también coloniza chopos, nogales y olmos; puede habitar en agallas vacías (Bernard, 1968). Los nidos se sitúan sobre las ramas más altas. Es muy común en toda la región mediterránea.

Pese a no tener datos concretos al respecto, pensamos que se puede alimentar en parte de la melaza de los pulgones. Se ha recogido sobre Juglans regia, al igual que ha ocurrido con otras especies arborícolas que hemos encontrado en este trabajo.

Panaphis juglandis Frisch, 1734: Juglans regia L. $(13: 11.7 .1991)$.

\section{Formicinae: Formicini}

\section{Formica cunicularia Latreille, 1798}

Es una especie que anida bajo piedras o en terraplenes de tierra, en sociedades no muy numerosas, $\mathrm{y}$ es principalmente predadora o carroñera. Distribuida por Europa y Asia, es común en el occidente europeo.

Aphis (A.) fabae Scopoli, 1763: Cirsium arvense (L.) Scop. (8:11.7.1991). Aphis (A.) frangulae gossypii Glover, 1877: Scorzonera laciniata L. (19:26.6.1990). Aphis (A.) punicae (Passerini, 1863): Punica granatum L. (46:21.4.1988). Brachycaudus (Appelia) sp.: Prunus persica (L.) Batsch (1:8.11.1991). Cinara (C.) pectinatae (Nördlinger, 1880): Abies alba Miller (16:7.6.1990). Macrosiphoniella (M.) absinthii (L., 1758): Artemisia absinthium L. (8:29.5.1991). Macrosiphoniella (M.) artemisiae (Boyer de Fonscolombe, 1841): Artemisia absinthium L. (8:29.5.1991).

De las siete muestras que se han recogido de esta especie, en tres de ellas se encontraba junto a Lasius niger, atendiendo a sendas especies de pulgones de tres géneros diferentes: Aphis, Brachycaudus y Cinara.

\section{Formica fusca Linné, 1758}

Se alimenta de otros insectos, el néctar de las flores y la melaza de pulgones. Los hormigueros son muy populosos y se suelen localizar en bosques de pinos, de suelo principalmente calcáreo. Se distribuye ampliamente por toda la región holártica.

El óptimo de altitud, según Bernard (1968), se encuentra entre 1.000 y 1.600 metros, lo cual concuerda bastante bien con las altitudes de las dos localidades donde se ha encontrado esta especie en el presente estudio.

Cinara (C.) maritimae (Dufour, 1833): Pinus halepensis Miller (4:18.7.1991). Cinara (Cupressobium) juniperi (De Geer, 1773): Juniperus communis L. (4:27.6.1991). 
Se ha recogido junto a Formica subrufa, sobre Pinus halepensis, atendiendo a Cinara (C.) maritimae.

\section{Formica subrufa Roger, 1859}

Construye los nidos, de no muchos individuos, bajo las piedras, y vive en lugares no demasiado húmedos. Se trata de una especie de distribución fundamentalmente ibérica, pero que se puede encontrar en otros lugares, como Francia.

Aphis (A.) brotericola Mier, 1978: Euphorbia segetalis L. (4:13.7.1990/6.6.1991). Aphis (A.) fabae Scopoli, 1763: Digitalis obscura L. (4:27.6.1991). Cinara (C.) maritimae (Dufour, 1833): Pinus halepensis Miller (4:18.7.1991), Pinus nigra Arnold ssp. salzmannii (Dunal) Franco (4:3.7.1990). Thelaxes suberi (Del Guercio, 1911): Quercus ilex L. (33:5.7.1990).

Se ha encontrado junto a Formica fusca, sobre Pinus halepensis, atendiendo a Cinara (C.) maritimae, y junto a Tapinoma nigerrimum, atendiendo a Aphis (A.) brotericola, sobre Euphorbia segetalis (4:6.6.1991).

\section{Formicinae: Lasiini}

Lasius brunneus (Latreille, 1798)

Se diferencia esta especie de Lasius alienus (Förster, 1850) por presentar un muy claro surco frontal, así como triángulo frontal y ocelos. Vive sobre troncos muertos, a veces sobre madera viva, pero también bajo las piedras, prefiriendo los suelos silíceos. Busca pulgones, a los cuales llega a transportar, incluso a los de gran tamaño. Se distribuye principalmente por el centro y sur de Europa, si bien también hay citas de otras partes de Europa, el norte de África y Paquistán.

La alternativa Lasius alienus/Lasius brunneus, pese a las diferencias señaladas, no es muy clara en regiones meridionales como las de nuestra área de estudio. Sin embargo, a nuestro parecer, los ejemplares capturados deben asignarse a L. brunneus, ya que de las dos especies nos parecen más próximos a ésta.

Aphis (A.) fabae mordwilkoi Börner et Janisch, 1922: Pastinaca sativa L. ssp. sylvestris (Miller) Rouy et Camus (6:3.7.1990)

\section{Lasius emarginatus (Olivier, 1791)}

Los escapos y las tibias presentan abundantes pelos rígidos, anchamente espaciados los de los escapos antenales, y el tronco alar es rojo, en contraste con la cabeza y el gáster, que son oscuros. El aroma que desprende es muy fuerte, más penetran- te que el de las otras especies del género. Vive en lugares rocosos expuestos al sol y es de alimentación omnívora, prefiriendo atender pulgones, como ocurre en las otras especies de Lasius (Bernard, 1968). De origen europeo, se extiende también por Oriente Medio.

El comentario respecto a Lasius emarginatus/Lasius niger es similar al que hemos hecho para la especie anterior. L. emarginatus es problemática en regiones meridionales, mientras que $L$. niger es una especie muy común, de amplia distribución mundial. Si bien prácticamente todas las muestras nos parecen de esta última especie, las dos que reseñamos a continuación creemos que se aproximan más a L. emarginatus.

Aphis (A.) ruborum (Börner, 1932): Rubus ulmifolius Schott (24:17.5.1989). Brachycaudus (Appelia) schwartzi (Börner, 1931): Prunus persica (L.) Batsch (13:21.8.1991).

\section{Lasius niger (Linné, 1758)}

A diferencia de la especie anterior, los escapos antenales se encuentran repletos de pelos y el cuerpo está casi uniformemente coloreado, siendo el tronco alar de color pardo rojizo oscuro a negro. Es capaz de vivir en lugares muy diversos, si bien parece ser más abundante en terrenos calcáreos que en silíceos. Se trata de una hormiga no muy agresiva y que guarda a los áfidos en su nido durante el invierno para reponerlos en las plantas en primavera (Bernard, 1968). Atiende tanto a pulgones que viven sobre las partes aéreas de las plantas como a especies que viven bajo tierra. Sakata (1994) ha encontrado que los individuos concretos de Lasius niger atienden, al menos durante unos días, siempre a la misma especie de pulgón ante dos opciones, bien sea una de las especies de pulgón o la otra. Muy común en zonas con asentamientos humanos, se distribuye por toda la región holártica.

En una ocasión una hormiga cogió a una hembra vivípara alada de Cinara (C.) maghrebica entre sus mandíbulas y se la llevó del lugar como respuesta a ser molestada con el pincel.

Anoecia (A.) corni (Fabricius, 1775): Echinochloa crusgalli (L.) Beauv (6:16.8.1991). Anoecia (A.) major Börner, 1950: Echinochloa crus-galli (L.) Beauv (6:16.8.1991). Aphis (A.) affinis Del Guercio, 1911: Mentha longifolia (L.) Hudson (13:25.6.1991). Aphis (A.) confusa Walker, 1849: Scabiosa atropurpurea L. (42:23.5.1991). Aphis (A.) craccivora Koch, 1854: Cytisus heterochrous Webb ex Colmeiro (35:28.5.1991), Genista scorpius (L.) DC. (8:25.6.1991), Medicago L. (6:19.6.1990), Medicago sativa L. (1:13.7.1990), Retama sphaerocarpa (L.) Boiss. (64:20.6.1991). Aphis (A.) crepidis (Börner, 1940): Crepis vesicaria L. ssp. haenseleri (Boiss. ex DC.) P. D. Sell (6:19.6.1990,64:21.5.1991). Aphis (A.) cytiso- 
rum Hartig, 1841: Genista scorpius (L.) DC. (8:11.7.1991). Aphis (A.) fabae Scopoli, 1763: Arctium minus (Hill) Bernh (1:13.7.1990/27.6.1991,3:18.7.1991,6:2.8.1989/19.6.1990/3.7. 1990/13.7.1990), Beta vulgaris L. (6:18.7.1991), Carduus pycnocephalus L. (7:11.6.1991), Carduus tenuiflorus Curtis (16:7.6.1990), Centaurea calcitrapa L. (8:28.6.1990), Cirsium arvense (L.) Scop. (8:11.7.1991), Cirsium monspessulanum (L.) Hill. ssp. ferox (Cosson) Talavera (13:11.7.1991, 14:17.7.1991,42:23.5.1991), Cirsium pyrenaicum (Jacq.) All. (1:19.6.1990), Daucus carota L. (8:11.7.1991), Genista scorpius (L.) DC. (8:11.7.1991), Rubia peregrina L. (64:20.6.1991), Rumex conglomeratus Murray (42:12.6.1990), Rumex crispus L. (13:25.6.1991), Rumex sp. (1:8.11.1991, 65:.5.7.1990), Sonchus asper (L.) Hill (6:3.7.1990), Vicia faba L. (67:5.5.1991). Aphis (A.) fabae fabae Scopoli, 1763: Galium setaceum Lam. (42:23.5.1991), Papaver rhoeas L. (32:28.5.1991), Rumex pulcher L. (70:3.4.1990), Rumex sp. (6:6.6.1991,16:9.5.1991,64:21.5.1991), Vicia faba L. (56:24.4.1991). Aphis (A.) fabae solanella Theobald, 1914: Onopordum acanthium L. (1:6.6.1991,8:25.6.1991), Rumex crispus L. (35:28.5.1991). Aphis (A.) frangulae frangulae Kaltenbach, 1845: Cirsium monspessulanum (L.) Hill. ssp. ferox (Cosson) Talavera (42:23.5.1991). Aphis (A.) frangulae gossypii Glover, 1877: Labiatae (6:6.6.1991), Potentilla reptans L. (64:21.5.1991), Rosmarinus officinalis L. (60:21.5.1991), Solanum tuberosum L. (56:24.5.1989), Sonchus oleraceus L. (73:22.5.1990). Aphis (A.) helianthemi Ferrari, 1872: Helianthemum marifolium (L.) Miller (42:23.5.1991). Aphis (A.) intybi Koch, 1855: Cichorium intybus L. (1:3.7.1990, 14:26.6.1990). Aphis (A.) nasturtii Kaltenbach, 1843: Mentha longifolia (L.) Hudson (1:6.6.1991), Nasturtium officinale R. Br. (16:9.5.1991), Rumex crispus L. (35:28.5.1991), Rumex pulcher L. (74:22.5.1990). Aphis (A.) nepetae Kaltenbach, 1843: Nepeta nepetella L. ssp. cordifolia (Willk.) Ubera et Valdés (8:29.5.1991). Aphis (A.) psammophila Szelegiewicz, 1967: Dipsacus fullonum L. (6:27.6.1991). Aphis (A.) punicae (Passerini, 1863): Punica granatum L. (11:5.6.1990). Aphis (A.) ruborum (Börner, 1932): Rubus ulmifolius Schott (6:6.6.1991,8:21.8.1991,13:11.7.1991,16:9.5.1991, 24:29.5.1991, 35:28.5.1991, 64:14.6.1990). Aphis (A.) rumicis L., 1758: Rumex obtusifolius L. (1:19.9.1990), Rumex sp. (42:23.5.1991,64:21.5.1991). Aphis (A.) sambuci L., 1758: Sambucus ebulus L. (8:29.5.1991), Sambucus nigra L. (3:18.7.1991,8:25.6.1991/11.7.1991). Aphis (A.) sanguisorbae Schrank, 1801: Sanguisorba minor Scop. (35:28.5.1991). Aphis (A.) sedi Kaltenbach, 1843: Sedum album L. (8:25.6.1991,42:23.5.1991), Sedum sediforme (Jacq.) Pau (14:17.7.1991). Aphis (A.) sp.: Cirsium monspessulanum (L.) Hill. ssp. ferox (Cosson) Talavera (13:22.8.1990), Dipsacus fullonum L. (6:18.7.1991), Lavatera cretica L. (67:5.5.1991), Papaver rhoeas L. (42:23.5.1991). Aphis (A.) spiraecola Patch, 1914: Echium vulgare L. (46:14.4.1988), Eriobotrya japonica (Thunb.) Lindley (84:3.4.1990), Malus domestica Borkh. (13:11.7.1991), Pyracantha coccinea Roemer (58:7.10.1987), Pyrus communis L. (64:21.5.1991), Viburnum tinus L. (24:17.5.1989), Indeterminada (58:30.5.1987). Aphis (A.) umbrella (Börner, 1950): Lavatera cretica L. (42:12.6.1990), Malva neglecta Wallr. (1:27.6.1991, 6:3.7.1990/13.7.1990), Malva nicaeensis All. (64:21.5.1991). Aphis (A.) urticata Gmelin, 1790: Urtica dioica L. (6:6.6.1991, 8:29.5.1991/25.6.1991). Aphis (A.) verbasci Schrank, 1801: Verbascum sinuatum L. (14:17.7.1991, 43:9.7.1991).
Aulacorthum (A.) solani (Kaltenbach, 1843): Celtis australis L. (64:21.5.1991). Brachycaudus (Acaudus) cardui (L., 1758): Carduus assoi (Willk.) Pau (8:28.6.1990), Carduus pycnocephalus L. (7:11.6.1991,24:29.5.1991,42:23.5.1991), Carduus tenuiflorus Curtis (6:19.6.1990,16:7.6.1990), Compositae (8:29.5.1991), Cynara cardunculus L. (59:14.6.1990), Echium vulgare L. (19:26.6.1990), Galactites tomentosa Moench (74:22.5.1990), Onopordum acanthium L. (1:13.7.1990/ 6.6.1991/27.6.1991,2:2.7.1991,6:3.7.1990), Senecio jacobaea L. (1:3.7.1990). Brachycaudus (Acaudus) malvae Shaposhnikov, 1964: Malva parviflora L. (1:6.6.1991). Brachycaudus (Acaudus) populi (Del Guercio, 1911): Silene vulgaris (Moench) Garcke (9:7.6.1990,64:21.5.1991). Brachycaudus (Acaudus) sp.: Galactites tomentosa Moench (80:3.5.1991). Brachycaudus (Appelia) prunicola (Kaltenbach, 1843): Tragopogon porrifolius L. (64:21.5.1991). Brachycaudus (Appelia) schwartzi (Börner, 1931): Prunus persica (L.) Batsch (14:26.6.1990,17:29.6.1990,64:20.6.1991). Brachycaudus (Appelia) sp.: Prunus persica (L.) Batsch (1:8.11.1991). Brachycaudus (Appelia) tragopogonis (Kalten-bach, 1843): Scorzonera angustifolia L. (59:14.6.1990). Brachycaudus (B.) helichrysi (Kaltenbach, 1843): Prunus avium L. (32:28.5.1991), Prunus domestica L. (5:2.7.1991). Chaitophorus leucomelas Koch, 1854: Populus nigra L. (1:6.6.1991/ 18.7.1991,8:29.5.1991/11.7.1991,14:26.6.1990/17.7.1991,56: 2.4.1991,58:4.7.1991,64:14.6.1990/21.5.1991/20.6.1991). Chaitophorus populeti (Panzer, 1801): Populus alba L. (1:13.7.1990,8:28.6.1990/22.8.1990/11.7.1991,13:21.8.1991, 14:26.6.1990,16:7.6.1990,23:17.6.1990,60:21.5.1991). Chaitophorus populialbae (Boyer de Fons-colombe, 1841): Populus alba L. (35:28.5.1991,60:20.6.1991). Chaitophorus sp.: Populus alba L. (35:7.6.1990). Chromaphis juglandicola (Kaltenbach, 1843): Juglans regia L. (11:5.6.1990). Cinara (C.) maghrebica Mimeur, 1934: Pinus halepensis Miller (9:7.6.1990,16:7.6.1990,30:18.4.1991,35:28.5.1991,56:2.4.19 91,60:14.6.1990/21.5.1991). Cinara (C.) maritimae (Dufour, 1833): Pinus sylvestris L. (5:16.8.1991). Cinara (C.) pectinatae (Nördlinger, 1880): Abies alba Miller (16:7.6.1990). Cinara sp.: Pinus halepensis Miller (12:24.5.1990). Dysaphis (D.) apiifolia petroselini (Börner, 1950): Foeniculum vulgare Miller ssp. piperitum (Ucria) Coutinho (9:9.5.1991,65:18.6. 1991). Dysaphis (D.) meridialis Shaposhnikov, 1964: Rumex obtusifolius L. (1:19.9.1990). Dysaphis (Pomaphis) plantaginea (Passerini, 1860): Malus domestica Borkh. (6:6.6.1991, 32:24.5.1990/28.5.1991). Hyadaphis coriandri (Das, 1918): Daucus carota L. (8:11.7.1991). Hyadaphis foeniculi (Passerini, 1860): Daucus carota L. (8:11.7.1991). Hyalopterus pruni (Geoffroy, 1762): Arundo donax L. (60:14.6.1990,79:14.5.1991). Lachnus roboris (L., 1758): Quercus coccifera L. (6:3.7.1990), Quercus suber L. (24:18.6.1990). Lipaphis (L.) erysimi (Kal-tenbach, 1843): Nasturtium officinale R. Br. (16:9.5.1991). Macrosiphum (M.) euphorbiae (Thomas, 1878): Lepidium draba L. (27:30. 4.1991). Melanaphis donacis (Passerini, 1862): Arundo donax L. (40:10.7.1991,79:14.5.1991), Phragmites australis (Cav.) Trin ex Steudel (35:28.5.1991). Melanaphis pyraria (Passerini, 1861): Piptatherum miliaceum (L.) Cosson (24:17.5.1989), Pyrus communis L. (13:11.7.1991). Myzus (M.) cerasi (Fabricius, 1775): Prunus avium L. (1:27.6.1991, 6:16.8.1991, 32:28.5.1991, 40:10.7.1991). Myzus (Nectarosiphon) persicae (Sulzer, 1776): Celtis australis L. (64:21.5.1991), Lepidium draba L. (27:30.4.1991, 64:21.5.1991), Prunus persica (L.) 
Batsch (64:20.6.1991), Solanum tuberosum L. (56:24.5.1989). Nearctaphis bakeri (Cowen, 1895): Trifolium pratense L. (6:27.6.1991, 64:20.6.1991). Panaphis juglandis Frisch, 1734: Juglans regia L. (1:13.7.1990, 6:13.7.1990/18.7.1991/ 16.8.1991.13:21.8.1991). Pterocomma pilosum konoi Hori ex Takahashi, 1939: Salix babylonica L. (16:9.5.1991), Salix fragilis L. (1:6.6.1991). Pterocomma populeum (Kaltenbach, 1843): Populus alba L. (16:7.6.1990), Populus nigra L. (8:5.6.1990/19.9.1990,13:25.6.1991/11.7.1991,56:2.4.1991, 64:21.5.1991). Semiaphis dauci (Fabricius, 1775): Daucus carota L. (8:25.6.1991/11.7.1991). Sipha (Rungsia) maydis Passerini, 1860: Avena barbata Pott. ex Link (11:5.6.1990,42:12.6.1990), Lolium rigidum Gaudin (11:5. 6.1990). Indeterminado: Capsella bursa-pastoris (L.) Medicus (16:9.5.1991). Indeterminado: Cynara cardunculus L. (11:5.6. 1990). Indeterminado: Hordeum murinum L. (27:30.4.1991). Indeterminado: Juglans regia L. (6:2.8.1989). Indeterminado: Populus nigra L. (1:13.7.1990). Indeterminado: Punica granatum L. (65:18.6.1991). Indeterminado: Rubia peregrina L. (60:21.5.1991). Indeterminado: Rumex sp. (60:21.5.1991). Indeterminado: Salvia sp. (58:19.5.1988). Indeterminado: Vicia sativa L. (42:23.5.1991).

En tres ocasiones se ha encontrado junto a Formica cunicularia: atendiendo a Aphis (A.) fabae sobre Cirsium arvense, a Brachycaudus (Appelia) sp. sobre Prunus persica y a Cinara (C.) pectinatae sobre Abies alba. Se ha recogido, también, dos veces, junto a Tapinoma nigerrimum, ambas sobre Rumex crispus, una atendiendo a Aphis (A.) fabae solanella y la otra sobre Aphis (A.) nasturtii. En una muestra de Aphis (A.) frangulae gossypii, sobre Labiatae, se ha encontrado junto a Camponotus (Tanaemyrmex) aethiops, en otra de Aphis (A.) sedi, sobre Sedum album (42:3.5.1991), junto a Plagiolepis pygmaea, y en una última, se ha econtrado con otras tres especies de hormigas sobre Panaphis juglandis (6:16.8.1991): Crematogaster scutellaris, Dolichoderus (Hypoclinea) quadripunctatus y Camponotus (C.) fallax.

\section{Formicinae: Plagiolepidini}

\section{Plagiolepis pygmaea (Latreille, 1798)}

Es una hormiga muy agresiva, pese a su pequeño tamaño, que puede expulsar de su territorio a especies de mayor talla. Es omnívora, aunque tiene preferencia por los líquidos azucarados, por lo que las obreras transportan diversos homópteros hasta sus nidos, situados normalmente bajo las piedras. Se trata de una especie de distribución mediterránea.

Aphis (A.) brotericola Mier, 1978: Euphorbia segetalis L. (4:3.7.1990). Aphis (A.) cisticola Leclant et Remaudière, 1972: Cistus albidus L. (24:28.5.1990). Aphis (A.) craccivora Koch, 1854: Ceratonia siliqua L. (85:3.5.1991). Aphis (A.) fabae
Scopoli, 1763: Anethum graveolens L. (37:10.7.1991), Carduus assoi (Willk.) Pau (19:17.6.1990), Foeniculum vulgare Miller ssp. piperitum (Ucria) Coutinho (80:3.5.1991), Silybum marianum (L.) Gaertner (36:24.5.1990). Aphis (A.) fabae fabae Scopoli, 1763: Eryngium campestre L. (24:29.5.1991), Foeniculum vulgare Miller ssp. piperitum (Ucria) Coutinho (24:18.6.1990). Aphis (A.) fabae solanella Theobald, 1914: Solanum nigrum L. (24:30.10.1990). Aphis (A.) frangulae gossypii Glover, 1877: Anacyclus valentinus $\mathrm{L}$. (24:8.6.1989), Centaurium quadrifolium (L.) G. López et Jarvis ssp. barrelieri (Dufour) G. López (24:18.6.1990), Helichrysum stoechas (L.) Moench (24:18.6.1990), Potentilla reptans L. (84:3.4.1990), Psoralea bituminosa L. (24:17.5.1989). Aphis (A.) helianthemi Ferrari, 1872: Helianthemum syriacum (Jacq.) Dum.-Cours. (14:26.6.1990). Aphis (A.) sedi Kaltenbach, 1843: Sedum album L. (42:23.5.1991). Aphis (A.) sp., 1758: Ulex parviflorus Pourret (41:12.6.1990). Aphis (A.) sp.1: Rosmarinus officinalis L. (9:9.5.1991,76:4.6.1991). Aphis (A.) sp.2: Sideritis angustifolia Lag. (10:17.5.1990). Aphis (A.) spiraecola Patch, 1914: Pallenis spinosa (L.) Cass (31:31.5.1990), Indeterminada (24:18.6.1990). Brachycaudus (Acaudus) cardui (L., 1758): Carduus tenuiflorus Curtis (31:31.5.1990). Brachycaudus (Appelia) tragopogonis (Kaltenbach, 1843): Scorzonera angustifolia L. (24:18.6.1990). Chaitophorus populeti (Panzer, 1801): Populus alba L. (23:17.6.1990). Dysaphis (D.) sp.: Foeniculum vulgare Miller ssp. piperitum (Ucria) Coutinho (80:3.5.1991). Hyadaphis foeniculi (Passerini, 1860): Anethum graveolens L. (37:10.7.1991). Lachnus roboris (L., 1758): Quercus coccifera L. (60:14.6.1990). Myzus (Nectarosiphon) persicae (Sulzer, 1776): Calystegia sepium (L.) R. Br. (20:3.5.1990). Nearctaphis bakeri (Cowen, 1895): Trifolium pratense L. (6:18.7.1991). Thelaxes suberi (Del Guercio, 1911): Quercus ilex L. (15:28.6.1990). Toxoptera aurantii (Boyer de Fonscolombe, 1856): Erica multiflora L. (10:17.5.1990,24:28.5.1990). Uroleucon (U.) cichorii (Koch, 1855): Crepis vesicaria L. ssp. haenseleri (Boiss. ex DC.) P. D. Sell (31:31.5.1990). Indeterminado: Digitalis obscura L. (4:27.6.1991). Indeterminado: Dorycnium pentaphyllum Scop. ssp. pentaphyllum (24:29.5.1991).

Se ha encontrado junto a Camponotus (Myrmentoma) lateralis, atendiendo a Nearctaphis bakeri, junto a Lasius niger, atendiendo a Aphis (A.) sedi y, en dos ocasiones, junto a Crematogaster sordidula, una atendiendo a Aphis (A.) sp.1 (76:4.6.1991) y la otra atendiendo a Toxoptera aurantii (10:17.5.1990).

\section{Plagiolepis schmitzii Forel, 1895}

Vive bajo las piedras, sobre todo en lugares húmedos, muy a menudo cerca de cursos de agua, lo que es más raro en Plagiolepis pygmaea. Es una especie menos común que la anterior, que se da principalmente en el sur de Europa y el norte de África.

Aphis (A.) chloris Koch, 1854: Hypericum perforatum L. (11:5.6.1990,24:21.6.1989). Aphis (A.) craccivora Koch, 1854: Medicago sativa L. (8:5.6.1990). Aphis (A.) multiflorae Barbagallo et Stroyan, 1980: Erica multiflora L. (76:14.5. 
1991). Dysaphis (D.) sp.: Foeniculum vulgare Miller ssp. piperitum (Ucria) Coutinho (61:5.7.1990). Sipha (Rungsia) maydis Passerini, 1860: Bromus rubens L. (51:22.4.1990). Indeterminado: Crataegus monogyna Jacq. ssp. brevispina (G. Kunze) Franco (80:3.5.1991).

Se ha encontrado junto a Pheidole pallidula, atendiendo a Aphis (A.) chloris (11:5.6.1990), y junto a Tetramorium caespitum, atendiendo a Dysaphis sp.

\section{Myrmicinae: Crematogastrini}

\section{Crematogaster auberti Emery, 1869}

Es una hormiga hidrófila que construye sus nidos en suelos arcillosos, y de actividad nocturna principalmente. De origen norteafricano, se distribuye por el oeste de la región mediterránea.

Aphis (A.) brotericola Mier, 1978: Euphorbia segetalis L. (18:5.6.1990). Aphis (A.) craccivora Koch, 1854: Anthyllis cytisoides L. (60:20.6.1991). Aphis (A.) ruborum (Börner, 1932): Rubus ulmifolius Schott (14:17.7.1991). Aphis (A.) sp.: Helianthemum hirtum (L.) Miller (33:24.5.1990), Pallenis spinosa (L.) Cass (60:20.6.1991). Cinara (C.) maritimae (Dufour, 1833): Pinus halepensis Miller (4:16.8.1991), Pinus nigra Arnold ssp. salzmannii (Dunal) Franco (4:16.8.1991). Melanaphis donacis (Passerini, 1862): Arundo donax L. (60:20.6.1991).

\section{Crematogaster scutellaris (Olivier, 1791)}

Se trata de una especie típicamente arborícola (Tinaut, 1991). En este estudio se ha encontrado sobre árboles y arbustos en todas las ocasiones, excepto en una en la que se ha recogido sobre el ombligo de Venus. Es una hormiga agresiva que busca intensamente áfidos y cóccidos por la melaza de éstos. Construye sus nidos y galerías en el corcho, pudiendo ser muy perjudicial económicamente en la industria del corcho. Se trata de una especie muy común en la región mediterránea.

En una ocasión se comprobó que una de las hormigas estaba transportando una ninfa de Panaphis juglandis, a la que aparentemente se estaba llevando de la colonia de áfidos.

Aphis (A.) spiraecola Patch, 1914: Viburnum tinus L. (24:29.5.1991). Cinara (C.) maghrebica Mimeur, 1934: Pinus halepensis Miller (19:17.6.1990). Dysaphis (Pomaphis) plantaginea (Passerini, 1860): Malus domestica Borkh. (9:9.5.1991). Panaphis juglandis Frisch, 1734: Juglans regia L. (6:16.8.1991,14:26.6.1990). Thelaxes suberi (Del Guercio, 1911): Quercus ilex L. (24:28.5.1990). Indeterminado: Viburnum tinus L. (24:28.5.1990).

Junto a otras tres especies, Dolichoderus (Hypoclinea) quadripunctatus, Camponotus (C.) fallax y Lasius niger, se ha encontrado atendiendo a Panaphis juglandis (6:16.8.1991).

\section{Crematogaster sordidula (Nylander, 1848)}

Vive en lugares pedregosos y soleados, sobre todo silíceos, y a veces calcáreos. Es bastante común en la mayor parte de la región mediterránea.

Aphis (A.) craccivora Koch, 1854: Argyrolobium zanonii (Turra) P. W. Ball (15:25.6.1991). Aphis (A.) fabae Scopoli, 1763: Echinops ritro L. (37:10.7.1991), Sedum sediforme (Jacq.) Pau (76:4.6.1991). Aphis (A.) frangulae gossypii Glover, 1877: Rosmarinus officinalis L. (47:4.4.1991). Aphis (A.) multiflorae Barbagallo et Stroyan, 1980: Erica multiflora L. (76:4.6.1991). Aphis (A.) sedi Kaltenbach, 1843: Sedum sediforme (Jacq.) Pau (31:31.5.1990, 60:14.6.1990). Aphis (A.) serpylli Koch, 1854: Thymus vulgaris L. (60:21.5.1991). Aphis (A.) sp.: Ulex parviflorus Pourret (12:24.5.1990). Aphis (A.) sp.1: Rosmarinus officinalis L. (15:25.6.1991,76:4.6.1991). Brachycaudus (Acaudus) cardui (L., 1758): Carduus pycnocephalus L. (31:18.4.1991,81:15.5.1990). Toxoptera aurantii (Boyer de Fonscolombe, 1856): Erica multiflora L. (10:17.5.1990). Indeterminado: Ononis minutissima L. $(12: 24.5 .1990)$.

En dos ocasiones se ha encontrado compartiendo la colonia de pulgones con Plagiolepis pygmaea; concretamente sobre Aphis (A.) sp.1 (76:4.6.1991) y sobre la muestra de Toxoptera aurantii. También sobre Aphis (A.) sp.1, se ha encontrado junto a Tapinoma nigerrimum (15:25.6.1991).

\section{Myrmicinae: Myrmicini}

Myrmica scabrinodis Nylander, 1846

Se trata de una especie que construye nidos pequeños en hábitats variados, principalmente bajo las piedras, y que constituye sociedades poco numerosas. Es de distribución europea $\mathrm{y}$ asiática.

Aphis (A.) craccivora Koch, 1854: Genista scorpius (L.) DC. (4:27.6.1991). Cinara (C.) maritimae (Dufour, 1833): Pinus nigra Arnold ssp. salzmannii (Dunal) Franco (4:13.7.1990)

En las dos ocasiones en que se ha recogido esta especie, se ha encontrado junto a Camponotus (Myrmentoma) piceus. En el caso de Aphis (A.) craccivora, $C$. piceus era mucho más abundante.

\section{Myrmicinae: Pheidolini}

Pheidole pallidula (Nylander, 1848)

Es de alimentación omnívora, si bien existen razas especializadas (Bernard, 1968), ya que hay nidos donde sobre todo se encuentran granos, $\mathrm{y}$ 
otros en los que únicamente hay fragmentos de insectos. Parece ser originaria de Asia, extendiéndose en la actualidad por la región paleártica.

Aphis (A.) chloris Koch, 1854: Hypericum perforatum L. (11:5.6.1990). Aphis (A.) frangulae Kaltenbach, 1843: Veronica polita Fries (74:22.5.1990). Aphis (A.) sedi Kaltenbach, 1843: Umbilicus rupestris (Salisbury) Dandy (42:23.5.1991). Brachycaudus (Acaudus) cardui (L., 1758): Carduus tenuiflorus Curtis (10:17.5.1990). Nearctaphis bakeri (Cowen, 1895): Trifolium pratense L. (8:28.6.1990).

Se ha encontrado atendiendo a Aphis (A.) chloris junto a Plagiolepis schmitzii.

\section{Myrmicinae: Tetramoriini}

Tetramorium caespitum (Linné, 1758)

Se trata de una especie muy similar a Tetramorium semilaeve André, 1881, de la que se distingue difícilmente, si bien, según López (1991b), T. caespitum se puede separar de aquélla por un conjunto de caracteres, como el color más oscuro del cuerpo, tendente al negro, rugosidad más fuerte en la cabeza, el tórax, el peciolo y el postpeciolo, y, principalmente, por la forma del peciolo, que es globoso, con la cara posterior cayendo bruscamente hacia la inserción del postpeciolo, frente al peciolo aplastado de T. semilaeve, que cae inclinado hacia atrás. De actividad sobre todo nocturna, es una especie agresiva y con hormigueros muy poblados. Común como plaga de las casas, recoge semillas de los semilleros; roe tubérculos, raíces y tallos de varias plantas, y preda sobre otros artrópodos. Atiende también insectos que producen melaza (Krombein et al., 1979). Se encuentra distribuida por Eurasia, África y Norteamérica.

Aphis (A.) nerii Boyer de Fonscolombe, 1841: Nerium oleander L. (58:8.10.1987). Aphis (A.) sp.: Carthamus lanatus L. (6:18.7.1991). Dysaphis (D.) sp.: Foeniculum vulgare Miller ssp. piperitum (Ucria) Coutinho (61:5.7.1990).

Se ha encontrado junto a Plagiolepis schmitzii sobre Dysaphis sp.

Por último, mencionar que Messor capitatus (Latreille, 1798) ha sido recogida junto a una colonia de Brachycaudus (B.) helichrysi sobre Borago officinalis L. Sin embargo, se debe considerar que se trata de una coincidencia espacial, ya que las especies de este género son granívoras, casi estrictas. La costumbre de recoger granos de las flores hace muy probable su coincidencia con las colonias de áfidos.

\section{Comentarios y discusión}

Más que considerar relaciones estrictas entre especies de hormigas y especies de pulgones, podríamos decir que las hormigas tienen tendencia por determinados géneros, algunas de forma más estricta que otras. Pero en ocasiones hemos comprobado claramente que las relaciones dependían en primera instancia del lugar de recolección, donde predominaban más algunas especies de hormigas o incluso únicamente una de ellas.

Así, en la zona de la Albufera de Valencia (Devesa, El Palmar y Viveros Municipales de El Saler) únicamente hemos encontrado a Linepithema humile. En Jardines (Jardín Botánico de Valencia, Jardines del Real y Jardín del Campus de Burjassot), también hemos encontrado a esta hormiga como único representante de los formícidos que atienden a pulgones. Parece ser pues que esta especie desplaza a otras en las zonas citadas y es la única capaz, por tanto, de atender a los pulgones, habiendo sido encontrada junto a gran cantidad de especies de áfidos.

Otra especie, Myrmica scabrinodis, la hemos recogido en dos ocasiones en la provincia de Valencia, ambas en la misma localidad. En ambas la hemos encontrado junto a Camponotus (Myrmentoma) piceus, con la que comparte en nuestro estudio hábitats similares, por encima de los 700 metros de altura. Ambas, en conjunto, se han encontrado junto a Aphis (A.) craccivora, Aphis (A.) fabae, Cinara (C.) maritimae, Cinara (Cupressobium) cupressi y Cinara (Cupressobium) juniperi. Es fácil concluir que probablemente la no relación con otras especies de áfidos se debe más bien a que éstos se encuentren débilmente presentes en la zona o a que no puedan alcanzar dichas altitudes por ausencia o escasez de sus plantas nutricias, más que a la imposibilidad de ser atendidos por estas dos especies de hormigas, ya que lo que se observa es que, perteneciendo ambas a subfamilias diferentes, atienden a las mismas especies de pulgones, incluso a las mismas colonias, por coincidencia de hábitats.

Además de las zonas donde pueden desarrollarse determinadas especies, hemos de tener en cuenta los nichos ecológicos que poseen. Así, hemos recogido especies netamente arborícolas, que obviamente sólo podemos encontrar junto con pulgones que vivan exclusivamente en los árboles o al menos los tengan como hospedadores primarios. Así, por ejemplo, de las cinco muestras en las que hemos podido determinar la especie de áfido que se encontraba junto a Crematogaster scutellaris, cada 
una de ellas correspondía a un género diferente (Aphis, Cinara, Dysaphis, Panaphis y Thelaxes), pertenecientes estas cinco especies a cuatro subfamilias distintas. Las cinco especies típicamente arborícolas que hemos recogido en este estudio, Crematogaster scutellaris, Dolichoderus (Hypoclinea) quadripunctatus, Camponotus (C.) fallax, Camponotus (Myrmentoma) lateralis y Colobopsis truncatus, las hemos encontrado todas, entre otros pulgones, junto a Panaphis juglandis sobre Juglans regia; en una ocasión, las tres primeras en un mismo árbol. A este respecto, Tinaut (1991), en su estudio de los formícidos del Parque Nacional de Doñana, encontró a Crematogaster scutellaris, Camponotus lateralis y Colobopsis truncatus, normalmente siempre sobre alcornoques, aunque sin indicar si los encontró junto a pulgones; en nuestra provincia deben tener otros nichos, como los nogales, ya que los alcornoques son muy escasos y se encuentran en pequeños reductos. Es lícito pensar, pues, que las especies de pulgones que más veces podemos encontrar junto a estas hormigas son las características de los árboles más típicos de la zona, sin renunciar, pues, a pensar que en otros lugares sean otras las especies de pulgones atendidas.

Por otra parte, de las 26 especies de formícidos encontradas en este estudio en la provincia de Valencia, la comarca del Racó d'Ademús, muy peculiar dada su situación geográfica, se ha visto representada por 16 de dichas especies: Myrmica scabrinodis, Crematogaster auberti, Crematogaster scutellaris, Tetramorium caespitum, Dolichoderus (Hypoclinea) quadripunctatus, Tapinoma nigerrimum, Plagiolepis pygmaea, Lasius brunneus, Lasius niger, Camponotus (C.) fallax, Camponotus (Myrmetoma) lateralis, Camponotus (Myrmentoma) piceus, Camponotus (Tanaemyrmex) aethiops, Formica cunicularia, Formica fusca y Formica subrufa. De éstas, se han encontrado sólo en dicha comarca cinco de ellas: Myrmica scabrinodis, Dolichoderus (Hypoclinea) quadripunctatus, Lasius brunneus, Camponotus (C.) fallax y Formica fusca.

Teniendo en cuenta la altitud a la que se encuentra la localidad de menor altura que hemos muestreado en esta comarca (Ademuz, 741 metros), si establecemos un límite a los 700 metros de altitud, observaremos una serie de especies de hormigas que sólo hemos encontrado a partir de dicha altura. Además de las cinco que ya hemos mencionado, incluímos: Camponotus (Myrmentoma) piceus, Colobopsis truncatus y Formica subrufa.

Por encima de los 1000 metros de altitud, se encuentran, dentro de la provincia, 7 de las 85 loca- lidades muestreadas (Alpuente, Eras, Negrón, Puebla de San Miguel, El Rebollar, Sesga y La Yesa). Cuatro especies son las que únicamente hemos encontrado en estas alturas en el área de estudio: Myrmica scabrinodis, Colobopsis truncatus, Formica fusca y Formica subrufa.

En un enclave de especial importancia en la provincia de Valencia, como es el Monte de PortaCoeli, hemos encontrado las siguientes siete especies: Crematogaster scutellaris, Tapinoma nigerrimum, Plagiolepis pygmaea, Plagiolepis schmitzii, Lasius emarginatus, Lasius niger y Camponotus (Myrmosericus) cruentatus, esta última sólo la hemos encontrado en esta zona. Otra de las zonas interesantes de nuestra provincia, la Albufera y su Devesa, ha mostrado menor diversidad en cuanto a la fauna de formícidos que se encuentran junto a pulgones (únicamente Linepithema humile, como ya hemos comentado).

Asociadas exclusiva o fundamentalmente con árboles y arbustos en la provincia, hemos encontrado a 7 de las 26 especies que citamos: Myrmica scabrinodis, Crematogaster scutellaris, Dolichoderus (Hypoclinea) quadripunctatus, Camponotus (C.) fallax, Camponotus (Myrmentoma) piceus, Colobopsis truncatus y Formica fusca.

Todas las muestras de Aphis (A.) fabae que hemos encontrado sobre Arctium minus (un total de 7, todas recogidas en el Racó d'Ademús) tienen sólo a Lasius niger como hormiga que los atiende, pese a que en la zona hemos encontrado muchas de las especies de Formicidae que citamos en este trabajo, entre las cuales Lasius brunneus, Camponotus (Tanaemyrmex) aethiops y Formica subrufa se encontraban sobre Aphis (A.) fabae, si bien no sobre Arctium minus. Esto parece indicar una tendencia mayor de Lasius niger respecto a las otras especies hacia la planta (más que hacia el pulgón).

Por otra parte, las dos especies de áfidos que hemos encontrado junto a un mayor número de especies de hormigas son Aphis (A.) craccivora y Aphis (A.) fabae, que se han encontrado junto a 14 especies diferentes cada uno de ellos. Aphis (A.) frangulae y Brachycaudus (Acaudus) cardui se han encontrado ambas junto a 8 especies diferentes de hormigas, Cinara (C.) maritimae con 7 y junto a 6 especies distintas se ha encontrado a Panaphis juglandis y Thelaxes suberi.

Lasius niger es la hormiga que hemos encontrado junto a un mayor número de áfidos (58 especies diferentes). También Linepithema humile y Tapi-noma nigerrimum, y algo menos Plagiolepis pygmaea, se han encontrado con un gran número de especies distintas de pulgones $(31,30$ y 20 respectivamente). 
Cuando se trata de hacer comparaciones entre número de muestras con y sin hormigas, en ocasiones pueden no ser indicativos los datos obtenidos si pretendemos establecer la mirmecofilia o mirmecofobia de los áfidos, pues existen especies que se encuentran atendidas por las hormigas únicamente en uno de los dos hospedadores, el primario o el secundario. Por tanto, el no encontrar hormigas junto a las colonias de pulgones no nos indica necesariamente que el áfido sea mirmecófobo. Las especies de Anoecia son atendidas por hormigas únicamente en las raíces de las gramíneas y ciperáceas, y las de Tetraneura lo son sobre el hospedador secundario, ya que en el primario viven en agallas (Heie, 1980). Así, de las 11 muestras recogidas de pulgones del género Tetraneura, 10 de ellas lo han sido sobre Ulmus y sólo una sobre un hospedador secundario, precisamente en la que hemos recogido también hormigas. De los otros géneros de pulgones que hemos encontrado en agallas, Baizongia, Forda y Geoica son mirmecófilos (Roberti, 1983). Excepto en una ocasión en la que se ha encontrado a Forda formicaria accidental sobre Torilis nodosa, el resto de muestras las hemos recogido sobre agallas en Pistacia terebinthus y, debido a ello, pese a que no los hemos encontrado nunca junto a hormigas, no podemos afirmar que se trate de géneros mirmecófobos.

De las 227 especies de Aphididae recogidas sobre sus plantas hospedadoras en el estudio realizado, en 93 hemos encontrado hormigas, si bien algunas de ellas son dudosas como mirmecófilas.

Las especies que más veces hemos encontrado con hormigas y con mayor posibilidad de ser mirmecófilas corresponden al género Aphis (de 55 especies del subgénero Aphis que hemos recogido en este estudio, hemos encontrado junto a hormigas 37 de ellas). En otros géneros también hemos encontrado un elevado número de especies relacionadas de forma clara con las hormigas. Así, de las 6 especies de Chaitophorus encontradas en este trabajo en 3 de ellas se ha visto relación con las hormigas, las 3 que viven sobre Populus, mientras que en las 3 que hemos encontrado sobre Salix no hemos encontrado esta relación. Estos datos vienen apoyados por los que ya conocemos sobre las especies de este género (Heie, 1982; Blackman y Eastop, 1994), y parece interesante apuntar que las hormigas podrían estar discriminado los sauces a favor de una exploración de los chopos en busca de pulgones. En cuanto al género Cinara, de las 8 especies encontradas, en 6 hemos observado relación con las hormigas, tanto sobre Pinaceae como sobre Cupressaceae. De 10 especies de Brachy- caudus, en 8 de ellas hemos observado relación con las hormigas, si bien la especie del subgénero Brachycaudus parece comportarse como mirmecófoba. En cuanto a Dysaphis, de 9 especies recogidas, 4 de ellas las hemos encontrado junto a hormigas, tanto en plantas herbáceas como en árboles. Las 3 especies de Melanaphis representadas en este trabajo se encontraban en alguna ocasión junto a hormigas, tanto sobre gramíneas como sobre peral. También las dos especies de Pterocomma recogidas en este estudio se han encontrado junto a hormigas, siempre con Lasius niger, esta vez tanto sobre chopos como sobre sauces, a diferencia de lo que hemos encontrado respecto al género Chaitopho-rus. Es interesante señalar que las hormigas que fundamentalmente hemos encontrado con este género son Linepithema humile y otra vez Lasius niger, que como acabamos de comentar sí la hemos recogido sobre Salix, por lo que lo apuntado anteriormente sobre la preferencia de chopos sobre sauces tendríamos que analizarlo más profundamente.

\section{AGRADECIMIENTOS}

Hemos de agradecer informaciones interesantes y un apoyo fundamental en la determinación de algunas especies de pulgones a los Doctores Juan Manuel Nieto Nafría y Pilar Mier Durante de la Universidad de León, Antonio Meliá del Servicio de Sanidad Vegetal de Castellón, Fernando Albano Ilharco de la Estación Agronómica Nacional de Oeiras y Alfonso Hermoso del I.V.I.A. de Moncada (Valencia).

\section{Referencias}

Alsina, A., Cerdá, X., Retana, J. y Bosch, J., 1988. Foraging ecology of the aphid-tending ant Camponotus cruentatus (Hymenoptera, Formicidae) in a savanna-like grassland. Miscel.lània Zoològica, 12: $195-204$.

Bernard, F., 1968. Faune de l'Europe et du Bassin Méditerranéen 3: Les Fourmis (Hymenoptera Formicidae) d'Europe Occidentale et Septentrionale. Masson et Cie. Paris. 416 págs.

Blackman, R. L. y Eastop, V. F., 1994. Aphids on the World's Trees: An Identification and Information guide. CAB International. Wallingford (UK). 1.006 pp.

BRISTOw, C. M., 1991a. Are ant-aphid associations a tritrophic interaction? Olenader aphids and Argentine ants. Oecologia, 87: 514-521.

BRISTOW , C. M., 1991b. Why are so few aphids ant-tended? En: Cutler, D. F. y C. R. Husley (eds.). AntPlant Interaction, 9: 105-119. 
Collingwood, C. A., 1976. A provisional list of Iberian Formicidae with a key to the worker caste (Hym. Aculeata). Eos, 52: 65-95.

Delfino, M. A. y Buffa, L. M., 2000. Algunas interacciones planta-áfido-hormiga en Córdoba (Argentina). Zoologica Baetica, 11: 3-15.

Evans, H. C. y Leston, D., 1971. A Ponerinae ant associated with Homoptera on cocoa in Ghana. Bulletin of Entomological Research, 61: 577-583.

GONZÁlez FunES, P., 1987. Contribución al conocimiento de los pulgones (Homoptera, Aphidoidea) y de sus parasitoides (Hymenoptera, Aphidiidae) en la provincia de Alicante. Tesis doctoral. Universitat de València. 386 págs.

HeIE, O. E., 1980. The Aphidoidea (Hemiptera) of Fennoscandia and Denmark. I. General part. The families Mindaridae, Hormaphididae, Thelaxidae, Anoeciidae and Pemphigidae. Fauna entomologica Scandinavica, 9: 1-235.

HeIE , O. E., 1982. The Aphidoidea (Hemiptera) of Fennoscandia and Denmark. II. The family Drepanosiphidae. Fauna entomologica Scandinavica, 11: 1-175.

Hölldobler, B. y Wilson, E. O., 1990. The Ants. Belknap Press. Harvard University Press Cambridge. 732 págs.

Krombein, K. V., Hurd, JR., P. D., Smith, D. R. y Burks, B. D., 1979. Catalog of Hymenoptera in American North of Mexico. Volume 2. Smithsonian Institution Press. Washington, D. C. 1011 págs.

LóPEZ, F., 1991a. Estudio morfológico y taxonómico de los grupos de especies ibéricas del género Tetramorium Mayr, 1855 (Hym., Formicidae). Boletín de la Asociación española de Entomología, 15: 29-39.

LÓPEZ, F., 1991b. Variabilidad morfológica y problemas taxonómicos en Tetramorium caespitum (Linné, 1758) y Tetramorium semilaeve André, 1881 (Hym., Formicidae). Boletín de la Asociación española de Entomología, 15: 65-78.

Mier Durante, M. P., 1978. Estudio de la Afidofauna de la provincia de Zamora. Publicaciones Caja de Ahorros Provincial de Zamora. Zamora. 226 págs.

Nieto Nafría, J. M., Remaudière, G. y Mier Durante, M. P., 1991. Novedades para la afidofauna (Hom. Aphididae) española en la provincia fitogeográfica Orocantábrica (Cordillera Cantábrica, España). Boletín de la Asociación española de Entomología, 15: 317-324.

NúÑEZ PÉREZ, E., 1991. Bases para el desarrollo del control integrado de los pulgones (Hom. Aphididae) de los cultivos de la provincia de León. Tesis doctoral. Universidad de León. 341 págs.
Pontin, A. J., 1978. The numbers and distribution of subterranean aphids and their exploitation by the ant Lasius flavus. Ecological Entomology, 3: 203-207.

Retana, J., Bosch, J., Alsina, A. y Cerdá, X., 1987. Foraging ecology of the nectarivorous ant Camponotus foreli (Hymenoptera, Formicidae) in a savanna-like grassland. Miscel.lània Zoològica, 11: 187-193.

Roberti, D., 1983. Note su alcune specie di Fordinae (Homoptera, Aphidoidea, Eriosomatidae). Entomologica, 18: 151-214.

SAKATA, H., 1994. How an ant decides to prey on or to attend aphids. Research Population Ecology, 36(1): 45-51.

Suay Cano, V. A., 1996. Contribución al estudio de los pulgones (Homoptera: Aphididae) y sus parasitoides (Hymenoptera: Braconidae: Aphidiinae) en la provincia de Valencia. Tesis doctoral. Universitat de València. 469 págs.

SudD, J. H., 1987. Ant aphid mutualism. In: Mink, A. K. \& P. Harrewijn (eds.). Aphis their biology, natural enemies and control. Vol. A. Elsevier. Amsterdam: 355-365.

Tinaut, A., 1991. Contribución al conocimiento de los formícidos del Parque Nacional de Doñana (Hymenoptera, Formicidae). Boletín de la Asociación española de Entomología, 15: 57-63.

Tizado Morales, E. J., 1990. Estudio comparado de la fauna y la biología de los pulgones (Hom.), afidíinos (Hym.) y otros insectos acompañantes en dos áreas de la provincia de León. Tesis doctoral. Universidad de León. 294 págs.

Tizado, E. J., Tinaut, A. y Nieto Nafría, J. M., 1993. Relationships between ants and aphids in the province of León (Spain) (Hym: Formicidae; Hom: Aphididae). Vie et Milieu, 43(1): 63-68. 
Apéndice I. - Comarcas y localidades de muestreo.

Appendix I.- Sampling areas and localities.

\begin{tabular}{|c|c|c|c|c|c|}
\hline & Coordenadas & Altura & VII. L'HORTA & & \\
\hline I. EL RACÓ D'ADEMÚS & & & 44. Alboraia & 30 SYJ 27 & $6 \mathrm{~m}$ \\
\hline $\begin{array}{l}\text { 1. Ademuz } \\
\text { 1. }\end{array}$ & 30TXK43 & $741 \mathrm{~m}$ & 45. Alfafar & $30 \mathrm{SYJ} 26$ & $11 \mathrm{~m}$ \\
\hline 2. Mas del Olmo & 30 TXK53 & $990 \mathrm{~m}$ & 46. Burjassot & 30 SYJ 27 & $39 \mathrm{~m}$ \\
\hline 3. Negrón & 30TXK33 & $1027 \mathrm{~m}$ & 47. La Cañada & 30SYJ17 & $90 \mathrm{~m}$ \\
\hline 4. Puebla de San Miguel & 30TXK53 & $1100 \mathrm{~m}$ & 48. Catarroja & 30SYJ26 & $14 \mathrm{~m}$ \\
\hline 5. Sesga & 30 SXK53 & $1050 \mathrm{~m}$ & 49. Massanassa & 30 SYJ 26 & $12 \mathrm{~m}$ \\
\hline 6. Vallanca & $30 \mathrm{TXK} 43$ & $950 \mathrm{~m}$ & 50. Mislata & 30 SYJ 27 & $30 \mathrm{~m}$ \\
\hline II. ELS SERRANS & & & 51. Puçol & 30 SYJ38 & $15 \mathrm{~m}$ \\
\hline 11. ELS SERRANS & & & 52. El Palmar & 30SYJ35 & $13 \mathrm{~m}$ \\
\hline 7. Ahillas & $30 \mathrm{SXK} 70$ & $870 \mathrm{~m}$ & 53. Picanya & 30 SYJ 26 & $54 \mathrm{~m}$ \\
\hline 8. Alpuente & 30 SXK71 & $1002 \mathrm{~m}$ & 54. El Saler (Devesa) & 30 SYJ35 & $2 \mathrm{~m}$ \\
\hline 9. Benagéber & $30 \mathrm{SXJ} 69$ & $720 \mathrm{~m}$ & 55. Sedaví & $30 \mathrm{SYJ} 26$ & $11 \mathrm{~m}$ \\
\hline 10. Calles & 30 SXJ79 & $351 \mathrm{~m}$ & 56. Silla & 30 SYJ 26 & $8 \mathrm{~m}$ \\
\hline 11. Chelva & 30 SXK 70 & $474 \mathrm{~m}$ & 57. Torrent & 30 SYJ16 & $63 \mathrm{~m}$ \\
\hline 12. Chera & $30 \mathrm{SXJ} 78$ & $653 \mathrm{~m}$ & 58. València & 30 SYJ 27 & $5 \mathrm{~m}$ \\
\hline 13. Eras & 30SXK71 & $1000 \mathrm{~m}$ & VIII. LA VALL DE COFRENTS & & \\
\hline 14. Gestalgar & 30SXJ88 & $250 \mathrm{~m}$ & $\begin{array}{l}\text { 59. Ayora } \\
\text { 5. LA VALEN }\end{array}$ & 30 SXJ 62 & $598 \mathrm{~m}$ \\
\hline 15. Higueruelas & $30 \mathrm{SXK} 80$ & $870 \mathrm{~m}$ & 59. Ayora & $30 \mathrm{SXI} 64$ & $598 \mathrm{~m}$ \\
\hline 16. Tuéjar & 30 SXK 60 & $650 \mathrm{~m}$ & & 50SXJ04 & $394 \mathrm{~m}$ \\
\hline 17. Villar del Arzobispo & $30 \mathrm{SXK} 80$ & $520 \mathrm{~m}$ & 61. Cortes de Pallás & 30SXJ54 & $405 \mathrm{~m}$ \\
\hline 18. La Yesa & 30SXK71 & $1008 \mathrm{~m}$ & 62. Jalance & 30SXJ63 & $430 \mathrm{~m}$ \\
\hline III. EL CAMP DE TÚRIA & & & 63. Teresa de Cofrentes & $30 \mathrm{SXJ} 63$ & $533 \mathrm{~m}$ \\
\hline $\begin{array}{l}\text { 19. Alcublas } \\
\text { 19. EL A A }\end{array}$ & 30 SXK90 & $760 \mathrm{~m}$ & 64. Zarra & $30 \mathrm{SXJ} 62$ & $580 \mathrm{~m}$ \\
\hline 20. Benaguasil & $\begin{array}{l}\text { SUSANYO } \\
\text { 30SYJ08 }\end{array}$ & $108 \mathrm{~m}$ & IX. LA CANAL DE NAVARRÉS & & \\
\hline 21. Benissanó & $\begin{array}{l}30 \mathrm{~S} \text { IJ00 } \\
\text { 30SYJ08 }\end{array}$ & $102 \mathrm{~m}$ & 65. Anna & 30SYJ02 & $195 \mathrm{~m}$ \\
\hline 22. Bétera & 30SYJ18 & $92 \mathrm{~m}$ & 66. Bolbaite & 30SYJ02 & $241 \mathrm{~m}$ \\
\hline 23. Marines & 30 SYK 10 & $520 \mathrm{~m}$ & 67. Chella & 30SYJ02 & $200 \mathrm{~m}$ \\
\hline 24. Porta-Coeli & 30SYJ19 & $280 \mathrm{~m}$ & 68. Navarrés & 30SXJ93 & $275 \mathrm{~m}$ \\
\hline 25. Ribarroja de Túria & 30SYJ08 & $125 \mathrm{~m}$ & X. LA RIBERA ALTA & & \\
\hline 26. Sant Antoni de Benagéber & 30SYJ18 & $83 \mathrm{~m}$ & 69. Algemesí & $30 \mathrm{SYJ} 24$ & $18 \mathrm{~m}$ \\
\hline 27. Vilamarxant & 30SYJ08 & $160 \mathrm{~m}$ & XI. LA RIBERA BAIXA & & \\
\hline IV. EL CAMP DE MORVEDRE & & & 70. Cullera & 30SYJ33 & $3 \mathrm{~m}$ \\
\hline 28. Canet d'En Berenguer & 30 SYJ39 & $11 \mathrm{~m}$ & 71. Favareta & $30 \mathrm{SYJ} 33$ & $12 \mathrm{~m}$ \\
\hline 29. Gilet & 30SYJ29 & $76 \mathrm{~m}$ & 72. Polinyà del Xúquer & 30 SYJ24 & $7 \mathrm{~m}$ \\
\hline 30. Port de Sagunt & 30 SYJ39 & $11 \mathrm{~m}$ & XII. LA COSTERA & & \\
\hline 31. Sagunt & 30SYJ39 & $7 \mathrm{~m}$ & 73. Canals & 30SYJ01 & $133 \mathrm{~m}$ \\
\hline V. LA PLANA D'UTIEL & & & 74. Genovés & 30SYJ11 & $142 \mathrm{~m}$ \\
\hline 32. El Pontón & $30 \mathrm{SXJ} 67$ & $689 \mathrm{~m}$ & 75. Xàtiva & 30SYJ11 & $115 \mathrm{~m}$ \\
\hline 33. El Rebollar (Monte del Tejo) & $30 \mathrm{SXJ} 77$ & $1250 \mathrm{~m}$ & XIII. LA VALL D'ALBAIDA & & \\
\hline 34. Requena & $30 \mathrm{SXJ} 67$ & $692 \mathrm{~m}$ & 76. Albaida & $30 \mathrm{SYJ} 10$ & $323 \mathrm{~m}$ \\
\hline 35. Sinarcas & 30SXJ59 & $900 \mathrm{~m}$ & 77. Bocairent & 30SYH09 & $605 \mathrm{~m}$ \\
\hline 36. Utiel & $30 \mathrm{SXJ} 58$ & $820 \mathrm{~m}$ & 78. Carrícola & 30 SYJ 20 & $320 \mathrm{~m}$ \\
\hline VI. LA FOIA DE BUNYOL & & & 79. Montaverner & 30 SYJ10 & $200 \mathrm{~m}$ \\
\hline 37. Bunyol & 30 SXJ96 & $387 \mathrm{~m}$ & 80. Montixelvo & $30 \mathrm{SYJ} 20$ & $276 \mathrm{~m}$ \\
\hline 38. Chiva & 30SXJ97 & $240 \mathrm{~m}$ & 81. Ontinyent & 30SYH09 & $383 \mathrm{~m}$ \\
\hline 39. Godelleta & 30SXJ96 & $320 \mathrm{~m}$ & XIV. LA SAFOR & & \\
\hline 40. Hortunas & $30 \mathrm{SXJ} 76$ & $692 \mathrm{~m}$ & 82. Gandia & 30SYJ41 & $22 \mathrm{~m}$ \\
\hline 41. Macastre & 30SXJ96 & $430 \mathrm{~m}$ & 83. Ròtova & 30SYJ31 & $100 \mathrm{~m}$ \\
\hline 42. Siete Aguas & 30 SXJ77 & $700 \mathrm{~m}$ & 84. Xeraco & 30SYJ42 & $34 \mathrm{~m}$ \\
\hline 43. Yátova & 30 SXJ86 & $450 \mathrm{~m}$ & 85. Xeresa & 30SYJ42 & $29 \mathrm{~m}$ \\
\hline
\end{tabular}

Apéndice II.- Catálogo pulgón-hormiga. Se marcan con un asterisco $\left(^{*}\right)$ aquellas asociaciones que no se han encontrado descritas en la literatura consultada para España.

Appendix II.- Aphid-ant catalog. These associations that are not described in the literature consulted for Spain are indicated with an asterisk (*).

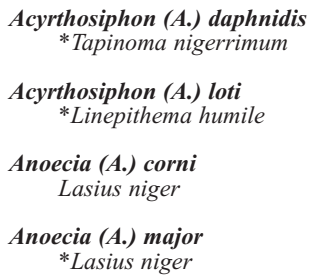

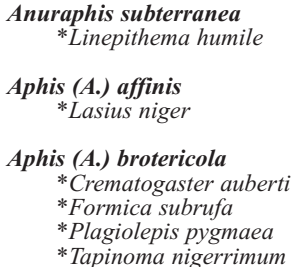

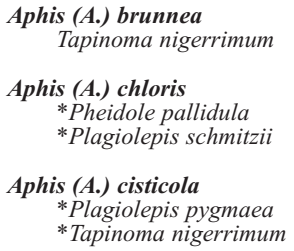




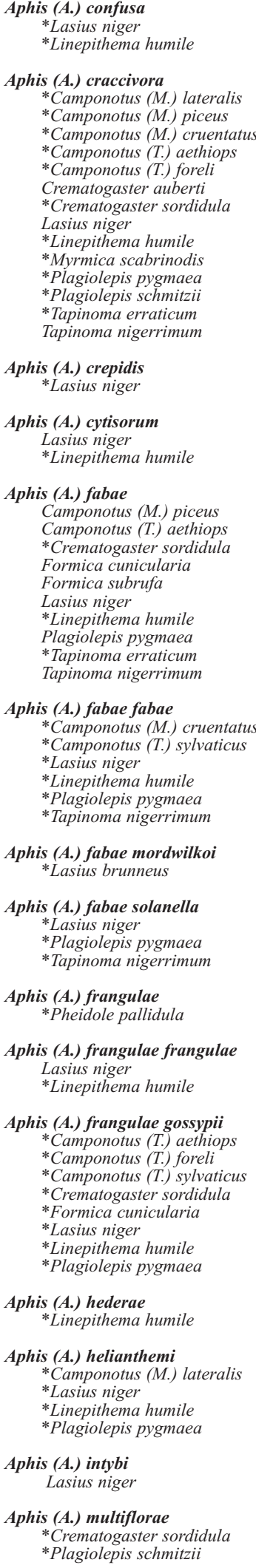

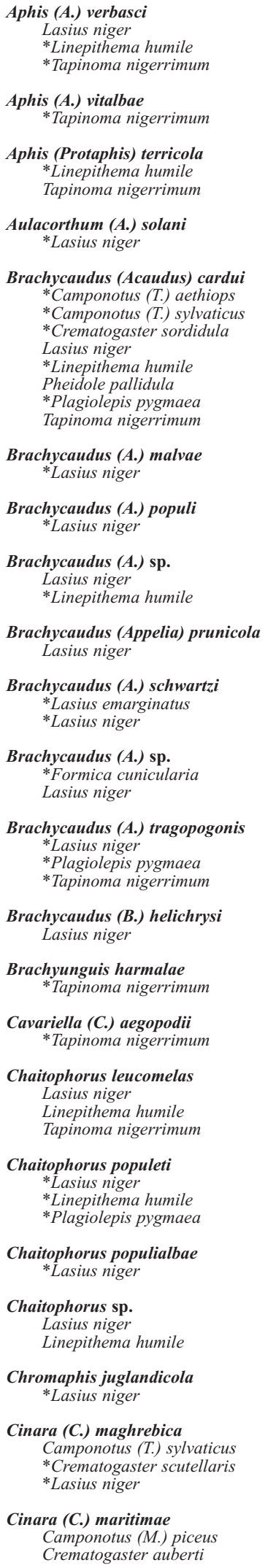

Brachyunguis harmalae *Tapinoma nigerrimum

Cavariella (C.) aegopodi * Tapinoma nigerrimum

Chaitophorus leucomelas Lasius niger Linepithema humile Tapinoma nigerrimum

Chaitophorus populeti * Lasius niger

*Linepithema humile *Plagiolepis pygmaea

Chaitophorus populialbae *Lasius niger

Chaitophorus sp. Lasius niger Linepithema humile

Chromaphis juglandicola *Lasius niger

Cinara (C.) maghrebica Camponotus (T.) sylvaticus Camponotus (T.) sylvaticus
$*$ Crematogaster scutellaris *Lasius niger

Cinara (C.) maritimae Camponotus (M.) piceus Crematogaster auberti 
Formica fusca

Formica subrufa

Lasius niger

*Myrmica scabrinodis

* Tapinoma nigerrimum

Cinara (C.) pectinatae

*Formica cunicularia *Lasius niger

Cinara (Cupressobium) cupress *Camponotus (M). piceus

Cinara (C.) juniperi *Camponotus (M.) piceus * Formica fusca * Tapinoma erraticum *Tapinoma nigerrimum

Cinara (C.) tujafilina *Linepithema humile *Tapinoma nigerrimum

Cinara sp.

Camponotus (T.) aethiops

Lasius niger

Tapinoma nigerrimum

Dysaphis (D.) apiifolia petroselini *Lasius niger

Tapinoma nigerrimum

Dysaphis (D.) meridialis

${ }^{*}$ Lasius niger

Dysaphis (D.) sp.

*Linepithema humile

*Plagiolepis pygmaea

Tetramorium caespitum

Dysaphis (D.) $\mathrm{sp1}$

Tapinoma nigerrimum

Dysaphis (Pomaphis) plantaginea

Crematogaster scutellaris

Lasius niger

* Tapinoma nigerrimum

Hyadaphis coriandri

*Lasius niger

\author{
Hyadaphis foeniculi \\ * Lasius niger \\ * Plagiolepis pygmaea \\ Hyalopterus pruni \\ Lasius niger \\ *Linepithema humile \\ Lachnus roboris \\ *Camponotus (T.) sylvaticus \\ * Lasius niger \\ * Plagiolepis pygmaea \\ Lipaphis (L.) erysimi \\ *Lasius niger \\ Macrosiphoniella (M.) absinthii \\ *Formica cunicularia \\ Macrosiphoniella (M.) artemisiae \\ *Formica cunicularia \\ Macrosiphoniella (M.) helichrysi \\ *Tapinoma nigerrimum \\ Macrosiphum (M.) euphorbiae \\ Lasius niger \\ *Linepithema humile \\ Melanaphis bambusae
*Linepithema hum \\ Melanaphis donacis \\ * Crematogaster auberti \\ * Lasius niger \\ *Linepithema humile \\ Melanaphis pyraria \\ Lasius niger \\ Myzocallis (M.) coryli
*Linepithema humile \\ us (M.) cerasi \\ Lasius niger \\ Myzus (Nectarosiphon) persicae \\ Lasius niger \\ *Plagiolepis pygmaea
}

Nearctaphis bakeri

*Camponotus (M.) lateralis

* Lasius niger

* Pheidole pallidula

*Plagiolepis pygmaea

Panaphis juglandis

*Camponotus (C.) fallax

*Camponotus (M.) laterali

*Colobopsis truncatu.

*Crematogaster scutellaris

* Dolichoderus (H.) quadripunctatus

Lasius niger

Pterocomma pilosum konoi *Lasius niger

Pterocomma populeum

${ }^{*}$ Lasius niger

Rhopalosiphum padi

*Linepithema humile

* Tapinoma nigerrimum

Semiaphis dauci

*Lasius niger

Sipha (Rungsia) maydis

*Lasius niger

*Plagiolepis schmitzii

*Tapinoma nigerrimum

Tetraneura (T.) ulmi

*Linepithema humile

Thelaxes suberi

*Crematogaster scutellaris

* Crematogaster sa

Linepithema humile

* Plagiolepis pygmaea

* Tapinoma nigerrimum

Toxoptera aurantii

*Crematogaster sordidula

*Linepithema humile

* Plagiolepis pygmaea

Uroleucon (U.) cichorii

*Plagiolepis pygmaea 